\title{
Unsupervised Discovery of Mid-Level Discriminative Patches
}

\author{
Saurabh Singh, Abhinav Gupta, and Alexei A. Efros \\ Carnegie Mellon University, Pittsburgh, PA 15213, USA \\ http://graphics.cs.cmu.edu/projects/discriminativePatches/
}

\begin{abstract}
The goal of this paper is to discover a set of discriminative patches which can serve as a fully unsupervised mid-level visual representation. The desired patches need to satisfy two requirements: 1) to be representative, they need to occur frequently enough in the visual world; 2) to be discriminative, they need to be different enough from the rest of the visual world. The patches could correspond to parts, objects, "visual phrases", etc. but are not restricted to be any one of them. We pose this as an unsupervised discriminative clustering problem on a huge dataset of image patches. We use an iterative procedure which alternates between clustering and training discriminative classifiers, while applying careful cross-validation at each step to prevent overfitting. The paper experimentally demonstrates the effectiveness of discriminative patches as an unsupervised mid-level visual representation, suggesting that it could be used in place of visual words for many tasks. Furthermore, discriminative patches can also be used in a supervised regime, such as scene classification, where they demonstrate state-of-the-art performance on the MIT Indoor-67 dataset.
\end{abstract}

\section{Introduction}

Consider the image in Figure11. Shown in green are the two most confident visual words 1 detected in this image and the corresponding visual word clusters. Shown in red are the two most confident detections using our proposed mid-level discriminative patches, computed on the same large, unlabeled image dataset as the visual words without any supervision. For most people, the representation at the top seems instantly more intuitive and reasonable. In this paper, we will show that it is also simple to compute, and offers very good discriminability, broad coverage, better purity, and improved performance compared to visual word features. Finally, we will also show how our approach can be used in a supervised setting, where it demonstrates state-of-the-art performance on scene classification, beating bag-of-words, spatial pyramids [2], ObjectBank [3], and scene deformable-parts models [4] on the MIT Indoor-67 dataset [5].

What are the right primitives for representing visual information? This is a question as old as the computer vision discipline itself, and is unlikely to be settled anytime soon. Over the years, researchers have proposed a plethora of different visual features spanning a wide spectrum, from very local to full-image,

A. Fitzgibbon et al. (Eds.): ECCV 2012, Part II, LNCS 7573, pp. 73-86, 2012.

(C) Springer-Verlag Berlin Heidelberg 2012 


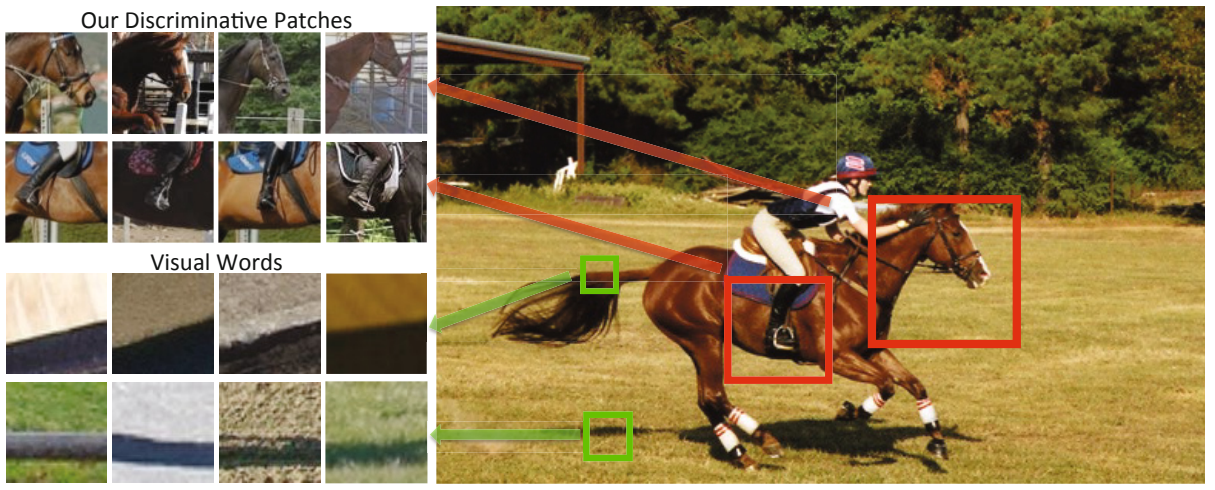

Fig. 1. The top two detected Visual Words (bottom) vs. Mid-level Discriminative Patches (top), trained without any supervision and on the same large unlabeled dataset

and from low-level (bottom-up) to semantic (top-down). In terms of spatial resolution, one extreme is using the pixel itself as a primitive. However there is generally not enough information at a pixel level to make a useful feature - it will fire all the time. At the other extreme, one can use the whole image as a primitive which, while showing great promise in some applications 67, requires extraordinarily large amounts of training data, since one needs to represent all possible spatial configurations of objects in the world explicitly. As a result, most researchers have converged on using features at an intermediate scale: that of an image patch.

But even if we fix the resolution of the primitive, there is still a wide range of choices to be made regarding what this primitive aims to represent. From the low-level, bottom-up point of view, an image patch simply represents the appearance at that point, either directly (with raw pixels [8]), or transformed into a different representation (filterbank response vector [9], blurred [1011] or spatially-binned [1213 feature, etc). At a slightly higher level, combining such patches together, typically by clustering and histogramming, allows one to represent texture information (e.g., textons [9], dense bag-of-words [2], etc). A bit higher still are approaches that encode image patches only at sparse interestpoints in a scale- and rotation-invariant way, such as in SIFT matching [12. Overall, the bottom-up approaches work very well for most problems involving exact instance matching, but their record for generalization, i.e. finding similar instances, is more mixed. One explanation is that at the low-level it is very hard to know which parts of the representation are the important ones, and which could be safely ignored.

As a result, recently some researchers have started looking at high-level features, which are already impregnated with semantic information needed to generalize well. For example, a number of papers have used full-blown object detectors, e.g. [14, as features to describe and reason about images (e.g. [15 3 16]). Others have employed discriminative part detectors such as poselets [17, attribute detectors [18], "visual phrases" [19], or "stuff" detectors [20] as features. 
However, there are significant practical barriers to the wide-spread adaptation of such top-down semantic techniques. First, they all require non-trivial amounts of hand-labeled training data per each semantic entity (object, part, attribute, etc). Second, many semantic entities are just not discriminative enough visually to act as good features. For example, "wall" is a well-defined semantic category (with plenty of training data available [21]), but it makes a lousy detector [21] simply because walls are usually plain and thus not easily discriminable.

In this paper, we consider mid-level visual primitives, which are more adaptable to the appearance distributions in the real world than the low-level features, but do not require the semantic grounding of the high-level entities. We propose a representation called mid-level discriminative patches. These patches could correspond to parts, objects, "visual phrases", etc. but are not restricted to be any one of them. What defines them is their representative and discriminative property: that is, that they can be detected in a large number of images with high recall and precision. But unlike other discriminative methods which are weakly supervised, either with image labels (e.g., 22]) or bounding-box labels (e.g., [14]), our discriminative patches can be discovered in a fully unsupervised manner given only a large pile of unlabeled images 1 . The key insight of this paper is to pose this as an unsupervised discriminative clustering problem on a huge unlabeled dataset of image patches. We use an iterative procedure which alternates between clustering and training discriminative classifiers (linear SVMs), while applying careful cross-validation at each step to prevent overfitting. Some of the resulting discriminative patches are shown in Figure 2.

Prior Work: Our goals are very much in common with prior work on finding good mid-level feature representations, most notably the original "visual words" approach [1. Given sparse key-point detections over a large dataset, the idea is to cluster them in SIFT space in an effort to yield meaningful common units of visual meaning, akin to words in text. However, in practice it turns out that while some visual words do capture high-level object parts, most others "end up encoding simple oriented bars and corners and might more appropriately be called 'visual phonemes' or even 'visual letters'." 24]. The way [24] addressed these shortcomings was by using image segments as a mid-level unit for finding commonality. Since then, there has been a large body of work in the general area of unsupervised object discovery [25|26|27|28|29|30|31. While we share some of the same conceptual goals, our work is quite different in that: 1) we do not explicitly aim to discover whole semantic units like objects or parts, 2) unlike [25/27/30], we do not assume a single object per image, 3 ) whereas in object discovery there is no separate training and test set, we explicitly aim to discover patches that are detectable in novel images. Because only visual words [1] have all the above properties, that will be our main point of comparison.

Our paper is very much inspired by poselets [17, both in its goal of finding representative yet discriminative regions, and its use of HOG descriptors and

\footnotetext{
${ }^{1}$ N.B.: The term "unsupervised" has changed its meaning over the years. E.g., while the award-winning 2003 paper of Fergus et al. 23] had "unsupervised" in its title, it would now be considered a weakly supervised method.
} 

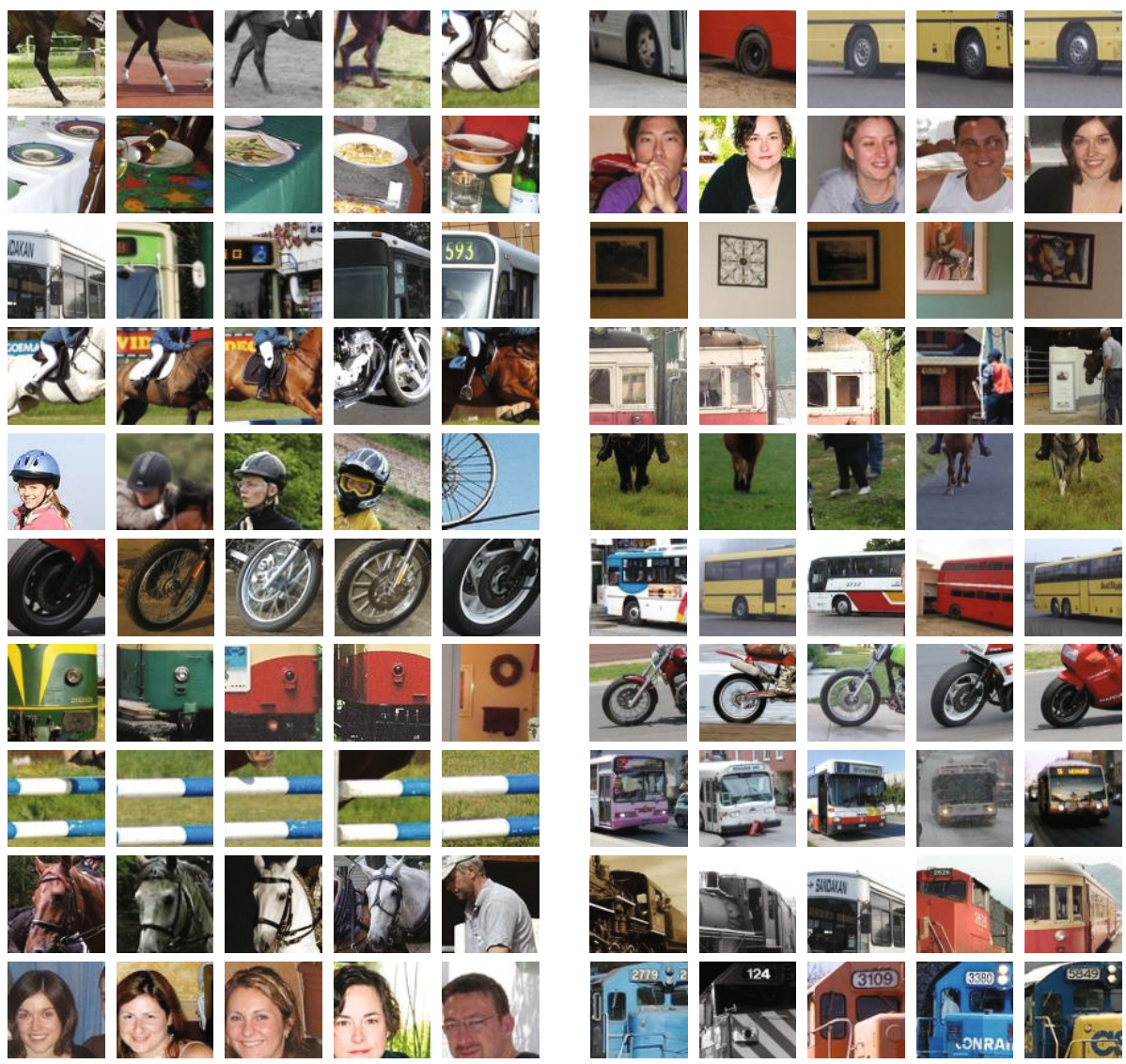

Fig. 2. Examples of discovered discriminative patches that were highly ranked

linear SVMs. However, poselets is a heavily-supervised method, employing labels at the image, bounding box, and part levels, whereas our approach aims to solve a much harder problem without any supervision at all, so direct comparisons between the two would not be meaningful. Our work is also informed by [32, who show that discriminative machinery, such as a linear SVM, could be successfully used in a fully unsupervised manner.

\section{Discovering Discriminative Patches}

Given an arbitrary set of unlabeled images (the "discovery dataset" $\mathcal{D}$ ), our goal is to discover a relatively small number of discriminative patches at arbitrary resolution which can capture the "essence" of that data. The challenge is that the space of potential patches (represented in this paper by HOG features [13]) is extremely large since even a single image can generate tens of thousands of patches at multiple scales. 


\subsection{Approach Motivation}

Of our two key requirements for good discriminative patches - to occur frequently, and to be sufficiently different from the rest of the visual world - the first one is actually common to most other object discovery approaches. The standard solution is to employ some form of unsupervised clustering, such as kmeans, either on the entire dataset or on a randomly sampled subset. However, running k-means on our mid-level patches does not produce very good clusters, as shown on Figure 3 (Initial KMeans). The reason is that unsupervised clustering like k-means has no choice but to use a low-level distance metric (e.g. Euclidean, $L 1$, cross-correlation) which does not work well for medium-sized patches, often combining instances which are in no way visually similar. Of course, if we somehow knew that a set of patches were visually similar, we could easily train a discriminative classifier, such as a linear SVM, to produce an appropriate similarity metric for these patches. It would seem we have a classic chicken-and-egg problem: the clustering of the patches depends on a good similarity, but learning a similarity depends on obtaining good clusters.

But notice that we can pose this problem as a type of iterative discriminative clustering. In a typical instantiation, e.g. [33], an initial clustering of data is followed by learning a discriminative classifier for each cluster. Based on the discriminatively-learned similarity, new cluster memberships can be computed by reassigning data points to each cluster, etc.. In principle, this procedure will satisfy both of our requirements: the clustering step will latch onto frequently occurring patches, while the classification step will make sure that the patches in the clusters are different enough from the rest, and thus discriminative. However, this approach will not work on our problem "as is" since it is infeasible to use a discovery dataset large enough to be representative of the entire visual world it will require too many clusters.

To address this, we turn the classification step of discriminative clustering into a detection step, making each patch cluster into a detector, trained (using a linear SVM) to find other patches like those it already owns. This means that each cluster is now trained to be discriminative not just against the other clusters in the discovery dataset $\mathcal{D}$, but against the rest of the visual world, which we propose to model by a "natural world dataset" $\mathcal{N}$. The only requirement of $\mathcal{N}$ is that it be very large (thousands of images, containing tens of millions of patches), and drawn from a reasonably random image distribution (we follow [32] in simply using random photos from the Internet). Note that $\mathcal{N}$ is not a "negative set", as it can (and most likely will) contain visual patterns also found in $\mathcal{D}$ (we also experimented with $\mathcal{D} \subset \mathcal{N})$.

It is interesting to note the similarity between this version of discriminative clustering and the root filter latent updates of [14]. There too, a cluster of patches (representing an object category) is being iteratively refined by making it more discriminative against millions of other image patches. However, whereas [14] imposes overlap constraints preventing the cluster from moving too far from the supervised initialization, in our unsupervised formulation the clusters are completely unconstrained. 


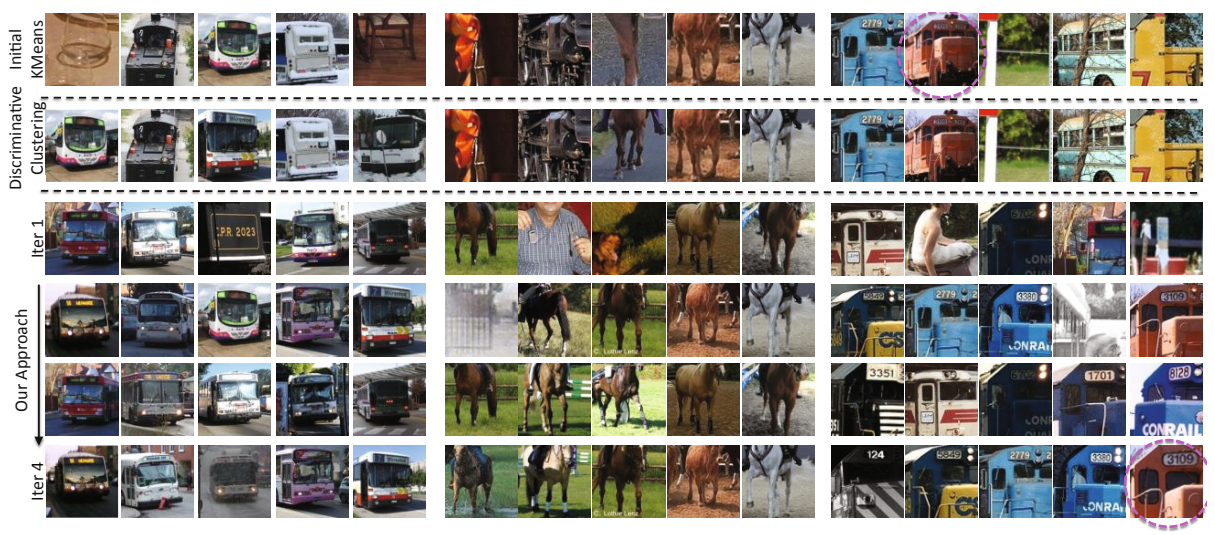

Fig. 3. Few examples to show how our iterative approach, starting with initial k-means clustering, converges to consistent clusters (Iter 4). While standard discriminative clustering approach (second row) also converges in some cases (1st column), in vast majority of cases it memorizes and overfits. Note that our approach allows clusters to move around in $\mathrm{x}, \mathrm{y}$ and scale space to find better members (Oval in 3rd column).

Alas, our proposed discriminative clustering procedure is still not quite enough. Consider Figure 3 which shows three example clusters: the top row is simple initialization using k-means, while the second row shows the results of the discriminative clustering described above. The left-most cluster shows good improvement compared to initialization, but the other two clusters see little change. The culprit seems to be the SVM - it is so good at "memorizing" the training data, that it is often unwilling to budge from the initial cluster configuration. To combat this, we propose an extremely simple but surprisingly effective solution - cross-validation training. Instead of training and classifying the same data, we divide our input dataset into two equal, non-overlapping subsets. We perform a step of discriminative clustering on the training subset, but then apply our learned discriminative patches on the validation subset to form clusters there. In this way, we are able to achieve better generalization since the errors in the training set are largely uncorrelated with errors in the validation set, and hence the SVM is not able to overfit to them. We then exchange the roles of training and validation, and repeat the whole process until convergence. Figure 3 shows the iterations of our algorithm for the three initial patch clusters (showing top 5 patches in each cluster). Note how the consistency of the clusters improves significantly after each iteration. Note also that the clusters can "move around" in $x, y$ and scale space to latch onto the more discriminative parts of the visual space (see the circled train in the right-most column).

\section{$2.2 \quad$ Approach Details}

Initialization: The input to our discovery algorithm is a "discovery dataset" $\mathcal{D}$ of unlabeled images as well as a much larger "natural world dataset" $\mathcal{N}$ (in this 


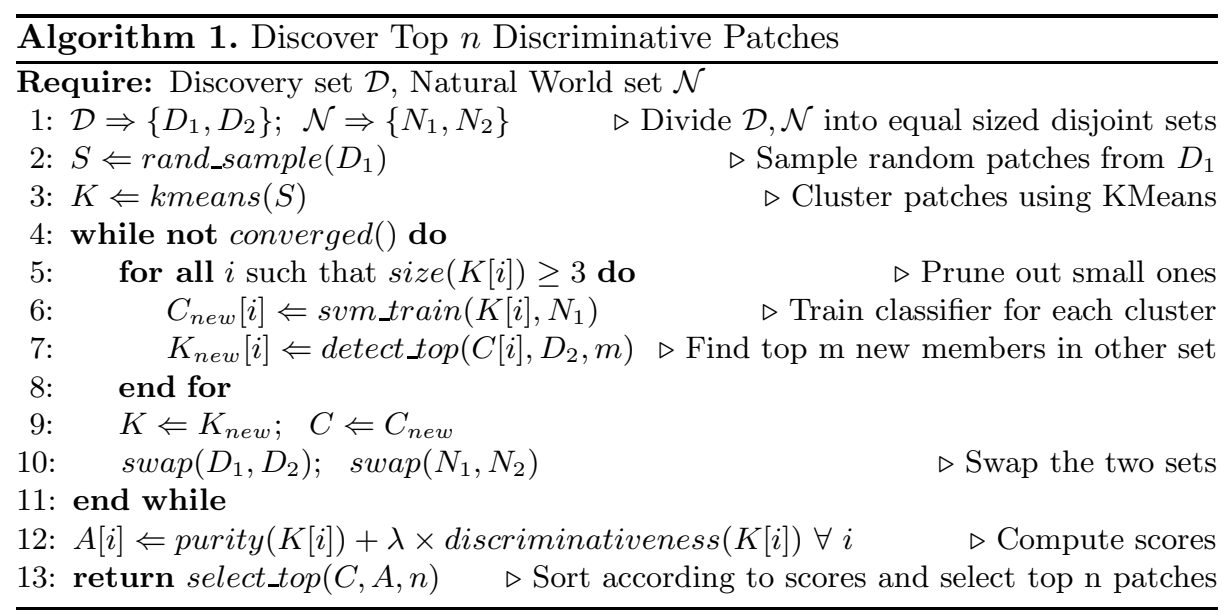

paper we used 6,000 images randomly sampled from Flickr.com). First, we divide both $\mathcal{D}$ and $\mathcal{N}$ into two equal, non-overlapping subsets $\left(D_{1}, N_{1}\right.$ and $\left.D_{2}, N_{2}\right)$ for cross-validation. For all images in $\mathcal{D}_{1}$, we compute HOG descriptors [13] at multiple resolutions (at 7 different scales). To initialize our algorithm, we randomly sample $S$ patches from $\mathcal{D}_{1}$ (about 150 per image), disallowing highly overlapping patches or patches with no gradient energy (e.g. sky patches) and then run standard $k$-means clustering in HOG space. Since we do not trust $k$-means to generalize well, we set $k$ quite high $(k=S / 4)$ producing tens of thousands of clusters, most with very few members. We remove clusters with less than 3 patches (eliminating $66 \%$ of the clusters), ending up with about 6 patches per image still active.

Iterative Algorithm: Given an initial set of clusters $K$, we train a linear SVM classifier [13] for each cluster, using patches within the cluster as positive examples and all patches of $N_{1}$ as negative examples (iterative hard mining is used to handle the complexity). If $D_{1} \subset N_{1}$, we exclude near-duplicates from $N_{1}$ by normalized cross-correlation $>0.4$. The trained discriminative classifiers are then run on the held-out validation set $D_{2}$, and new clusters are formed from the top $m$ firings of each detector (we consider all SVM scores above -1 to be firings). We limit the new clusters to only $m=5$ members to keep cluster purity high - using more produces much less homogeneous clusters. On the other hand, if a cluster/detector fires less than 2 times on the validation set, this suggests that it might not be very discriminative and is killed. The validation set now becomes the training set and the procedure is repeated until convergence (i.e. the top $m$ patches in a cluster do not change). In practice, the algorithm converges in 4-5 iterations. The full approach is summarized in Algorithm 1.

Parameters: The size of our HOG descriptor is $8 \times 8$ cells (with a stride of 8 pixels/cell), so the minimum possible patch is $80 \mathrm{x} 80$ pixels, while the maximum could be as large as full image. We use a linear SVM $(\mathrm{C}=0.1)$, with 12 iterations of hard negative mining. For more details, consult the source code on the website. 

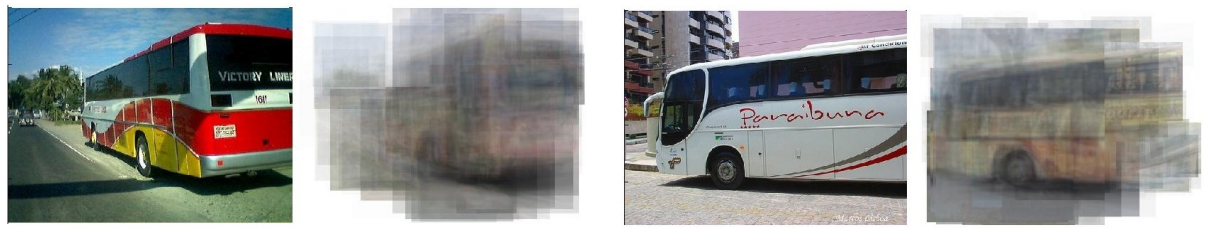

Fig. 4. Visualizing images (left) in terms of their most discriminative patches (right). The patch detectors were fired on a novel image and the high-scoring patch detections were averaged together, weighted by their scores.

\subsection{Ranking Discriminative Patches}

Our algorithm produces a dictionary of a few thousand discriminative patches of varying quality. Our next task is to rank them, to find a small number of the most discriminative ones. Our criteria for ranking consists of two terms:

Purity: Ideally, a good cluster should have all its member patches come from the same visual concept. However, measuring purity in an unsupervised setting is impossible. Therefore, we approximate the purity of each cluster in terms of the classifier confidence of the cluster members (assuming that cross-validation removed overfitting). Thus, the purity score for a cluster is computed by summing up the SVM detection scores of top $r$ cluster members (where $r>m$ to evaluate the generalization of the cluster beyond the $m$ training patches).

Discriminativeness: In an unsupervised setting, the only thing we can say is that a highly discriminative patch should fire rarely in the natural world. Therefore, we define discriminativeness of a patch as the ratio of the number of firings on $\mathcal{D}$ to the number of firings on $\mathcal{D} \cup \mathcal{N}$ (of course, we do not want patches that never fire at all, but these would have already been removed in cross-validation training).

All clusters are ranked using a linear combination of the above two scores. Figure 2 shows a set of top-ranked discriminative patch clusters discovered with our approach. Note how sometimes the patches correspond to object parts, such as "horse legs" and "horse muzzle", sometimes to whole objects, such as "plates", and sometimes they are just discriminative portions of an object, similar to poselets (e.g., see the corner of trains). Also note that they exhibit surprisingly good visual consistency for a fully unsupervised approach. The ability of discriminative patches to fire on visually similar image regions is further demonstrated in Figure 4 where the patch detectors are applied to a novel image and high-scoring detections are displayed with the average patch from that cluster. In a way, the figure shows what our representation captures about the image.

\section{Discovering "Doublets"}

While our discriminative patch discovery approach is able to produce a number of visually good, highly-ranked discriminative patches, some other potentially promising ones do not make it to the top due to low purity. This happens when 

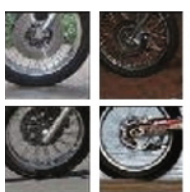

(a) Clean cluster
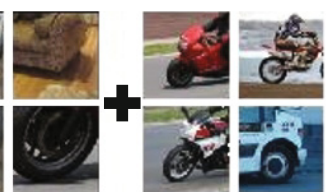

(b) Noisy Cluster
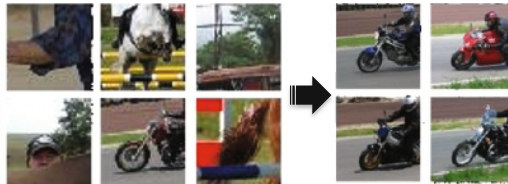

(c) Cleaned-up

Fig. 5. Cluster clean-up using "doublets". A visually non-homogeneous cluster (b) that has learned more than one concept, when coupled into a doublet with a high quality cluster (a), gets cleaned up (c).
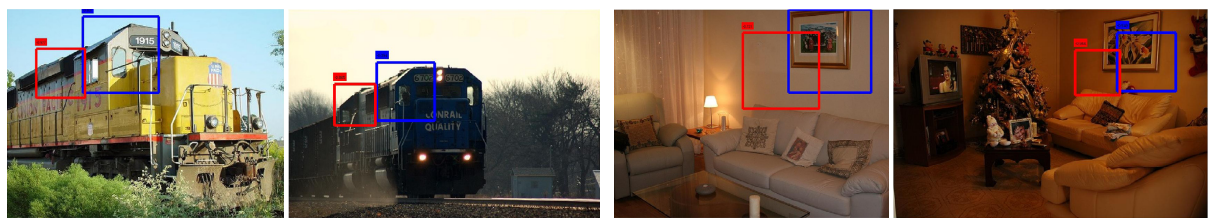

Fig. 6. Examples of discovered discriminative "doublets" that were highly ranked

a cluster converges to two or more "concepts" because the underlying classifier is able to generalize to both concepts simultaneously (e.g., Figure 5b). However, often the two concepts have different firing patterns with respect to some other mid-level patch in the dictionary, e.g., motorcycle wheel in Figure 5 a. Therefore, we propose to employ second-order spatial co-occurrence relationships among our discriminative patches as a way of "cleaning them up" (Figure 5r). Moreover, discovering these second-order relationships can provide us with "doublets" 34 (which could be further generalized to grouplets 22 35]) that can themselves be highly discriminative and useful as mid-level features in their own right.

To discover doublets, we start with a list of highly discriminative patches that will serve as high-quality "roots". For each root patch, we search over all the other discovered discriminative patches (even poor-quality ones), and record their relative spatial configuration in each image where they both fire. The pairs that exhibit a highly spatially-correlated firing pattern become potential doublets. We rank the doublets by applying them on the (unlabeled) validation set. The doublets are ranked high if in images where both patches fire, their relative spatial configuration is consistent with what was observed in the training set. In Figure 6 we show some examples of highly discriminative doublets. Notice that not only is the quality of discriminative patches good, but also the spatial relationships within the doublet are intuitive.

\section{Quantitative Evaluation}

As with other unsupervised discovery approaches, evaluation is difficult. We have shown a number of qualitative results (Figures 2, 6), and there are many more on the website. For the first set of quantitative evaluations (as well as for all the qualitative results except Figure 8), we have chosen a subset of of PASCAL VOC 2007 [36] (1,500 images) as our discovery dataset. We picked PASCAL 
VOC because it is a well-known and difficult dataset, with rich visual diversity and scene clutter. Moreover, it provides annotations for a number of object classes which could be used to evaluate our unsupervised discovery. However, since our discovered patches are not meant to correspond to semantic objects, this evaluation metric should be taken with quite a few grains of salt.

One way to evaluate the quality of our discriminative patch clusters is by using the standard unsupervised discovery measures of "purity" and "coverage" (e.g., 31]). Purity is defined by what percentage of cluster members correspond to the same visual entity. In our case, we will use PASCAL semantic category annotations as a surrogate for visual similarity. For each of the top 1000 discovered patches, we first assign it to one of the semantic categories using majority membership. We then measure purity as percentage of patches assigned to the same PASCAL semantic label. Coverage is defined as the number of images in the dataset "covered" (fired on) by a given cluster.

Figure 7 reports the purity and coverage of our approach and a number of baselines. For each one, the graphs show the cumulative purity/coverage as number of clusters being considered is increased (the clusters are sorted in the decreasing order of purity). We compare our approach with Visual Words [1] and Russell et. al 24 baseline, plus a number of intermediate results of our method: 1) HOG K-Means (visual word analog for HOG features), 2) Initial Clustering (SVMs trained on the K-Means clusters without discriminative re-clustering), and 3) No Cross-Validation (iterative, discriminatively-trained clusters but without cross-validation). In each case, the numbers indicate area-under-the-curve (AUC) for each method. Overall, our approach demonstrates substantial gain in purity without sacrificing much coverage as compared to the established approaches. Moreover, each step of our algorithm improves purity. Note in particular the substantial improvement afforded by the cross-validation training procedure compared to standard training.

As we mentioned, however, the experiment above under-reports the purity of our clusters, since semantic equivalence is not the same as visual similarity. Therefore, we performed an informal perceptual experiment with human subjects, measuring the visual purity of our clusters. We selected the top 30 clusters from the dataset. For each cluster, we asked human labelers to mark which of the cluster's top ten firings on the validation set are visually consistent with the cluster. Based on this measure, average visual purity for these clusters was $73 \%$.

\subsection{Supervised Image Classification}

Unsupervised clustering approaches, such as visual words, have long been used as features for supervised tasks, such as classification. In particular, bag of visual words and spatial pyramids 22 are some of the most popular current methods for image classification. Since our mid-level patches could be considered the true visual words (as opposed to "visual letters"), it makes sense to see how they would perform on a supervised classification task. We evaluate them in two different settings: 1) unsupervised discovery, supervised classification, and 2) supervised discovery, supervised classification. 

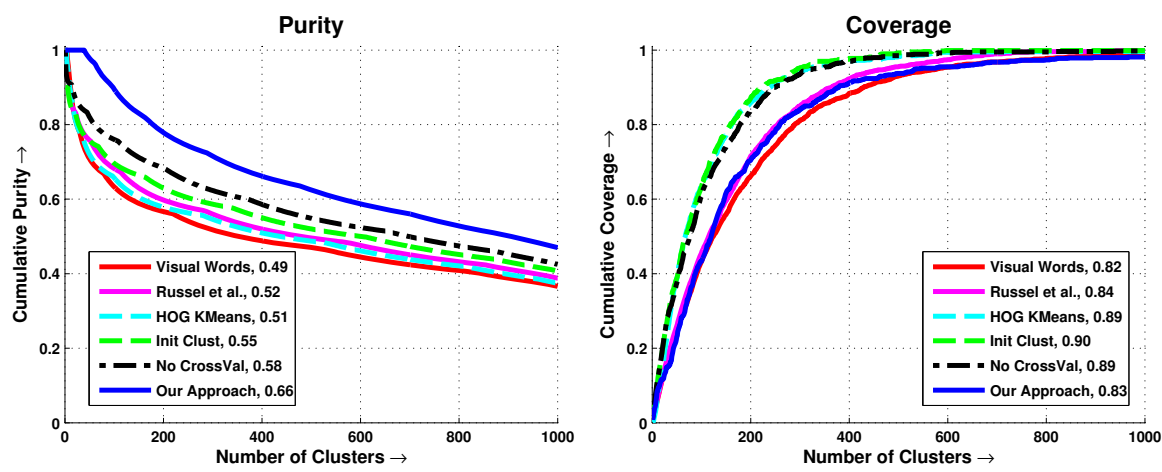

Fig. 7. Quantitative comparison of discriminative patches compared to the baseline approaches. Quality of clustering is evaluated in terms of the area under the curve for cumulative purity and coverage.

Table 1. Quantitative Evaluation: Average Classification on MIT Indoor-67 dataset. *Current state-of-the-art. †Best performance from various vocabulary sizes.

\begin{tabular}{|c|c|c|c|}
\hline GIST & 29.7 & Ours+GIST & 44.0 \\
\hline Spatial Pyramid HOG $(S P H O G)^{\dagger}$ & 29.8 & Ours +SP & 46.4 \\
\hline Spatial Pyramid SIFT $(S P)^{\dagger}$ & 34.4 & Ours+GIST + SP & 47.5 \\
\hline ROI-GIST [5] & 26.5 & Ours+DPM & 42.4 \\
\hline Scene DPM [4] & 30.4 & Ours+GIST+DPM & 46.9 \\
\hline MM-Scene 37. & 28.0 & Ours+SP+DPM & 46.4 \\
\hline Object Bank 3 & 37.6 & GIST+SP+DPM [4] & $43.1^{*}$ \\
\hline Ours & 38.1 & Ours+GIST+SP+DPM & 49.4 \\
\hline
\end{tabular}

\section{Unsupervised Discriminative Patches}

Using the discriminative patches discovered from the same PASCAL VOC discovery dataset as before, we would like to see if they could make better visual words for a supervised image classification task. Our baseline is the standard spatial pyramid of visual words (using 1000 visual words) using their public code [2] For our approach, we construct spatial pyramid using top 1000 discriminative patches. Classification was performed using a simple linear SVM and performance was evaluated using Average Precision. Standard visual words scored 0.54 AP, while using our discriminative patches, the score was $0.65 \mathrm{AP}$. We further expanded our feature representation by adding the top 250-ranking doublets as extra visual words, resulting in a slight improvement to $0.66 \mathrm{AP}$.

\section{Supervised Discriminative Patches}

We further want to evaluate the performance of our approach when it is allowed to utilize more supervision for a fair comparison with several existing supervised approaches. Instead of discovering the discriminative patches from a common pool of all the images, we can also discover them on a per-category basis. In this experiment, we perform supervised scene classification using the challenging MIT Indoor-67 dataset [5], containing 67 scene categories. Using the provided scene 

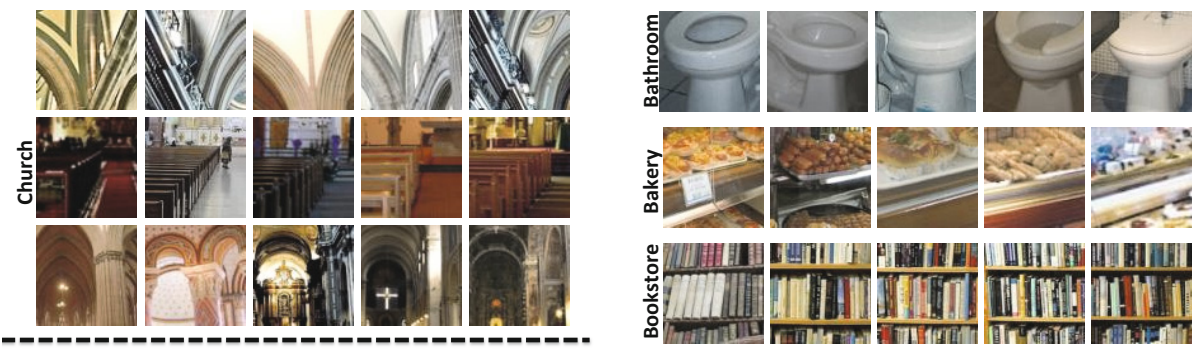

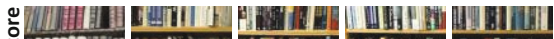
苟
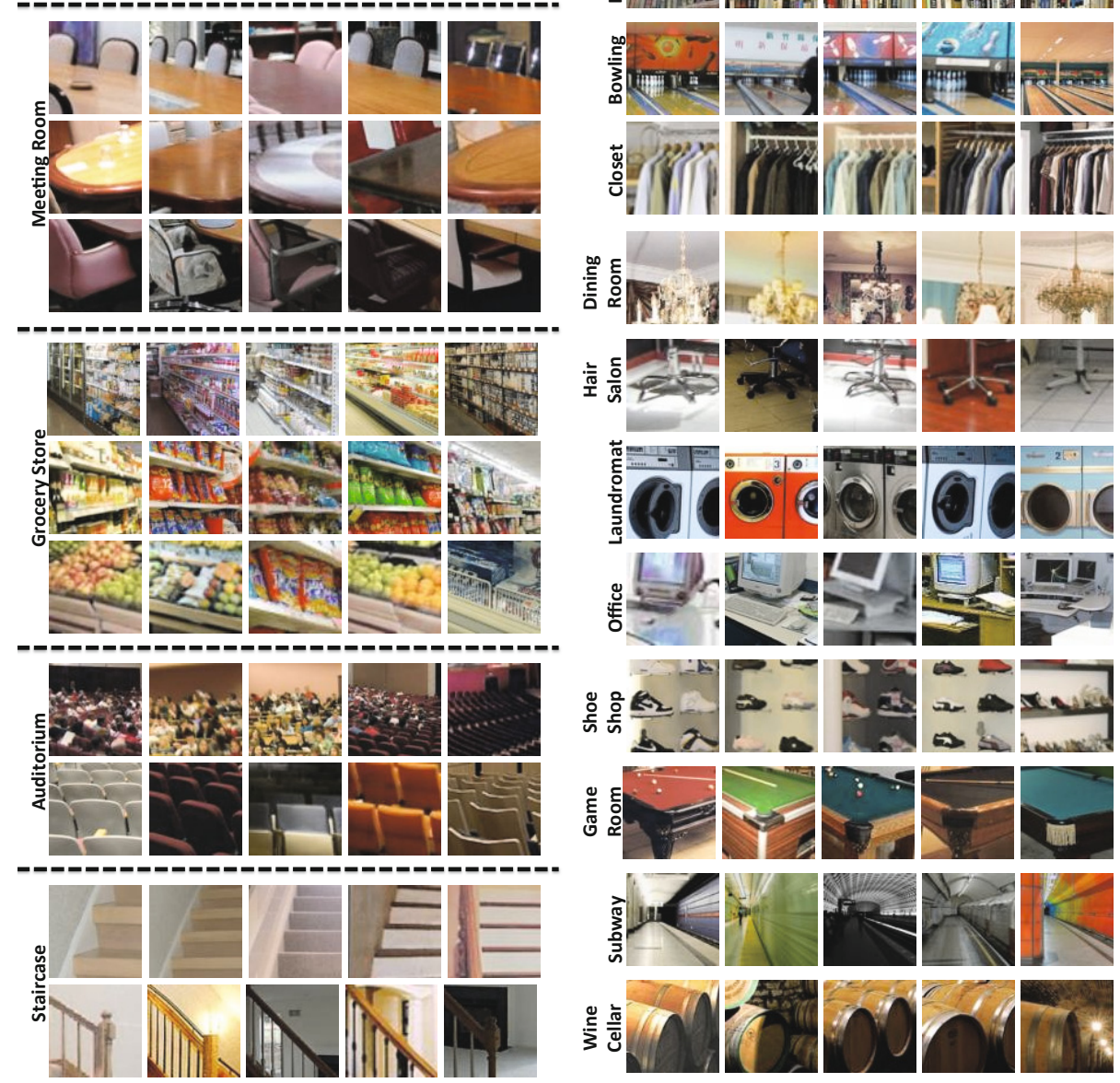

Fig. 8. Top discriminative patches for a sampling of scenes in the MIT Indoor-67 Scene dataset [5]. Note how these capture various visual aspects of a typical scene.

labels, we discover discriminative patches for each scene independently, while treating all other images in the dataset as the "natural world".

Figure 8 shows top few most discriminative patches discovered this way for a number of categories from the dataset. It is interesting to see that the discriminative patches capture aspects of scenes that seem very intuitive to us. In particular 
the discriminative patches for the Church category capture the arches and the benches; the ones for the Meeting Room capture the center table and the seats. These discriminative patches are therefore capturing the essence of the scene in terms of these highly consistent and repeating patterns and hence providing a simple yet highly effective mid-level representation. Inspired by these results, we have also applied a similar approach to discovering "What makes Paris look like Paris" 38] using geographic labels as the weak supervisory signal.

To perform classification, top 210 discovered patches of each scene are aggregated into a spatial pyramid using maxpooling over the discriminative patch scores as in [3]. We again use a linear SVM in a one-vs-all classification. The results are reported in Table 1. Comparison with HOG visual words (SPHOG) shows the huge performance gain resulting from our algorithm when operating in the same feature space. Further, our simple method by itself outperforms all others that have been tested on this dataset 5/4373]. Moreover, combining our method with the currently best-performing combination approach of 4 yields $\mathbf{4 9 . 4 \%}$ performance which, to our knowledge, is the best on this dataset.

Acknowledgments. The authors would like to thank Martial Hebert, Tomasz Malisiewicz, Abhinav Shrivastava and Carl Doersch for many helpful discussions. This work was supported by ONR Grant N000141010766.

\section{References}

1. Sivic, J., Zisserman, A.: Video Google: A text retrieval approach to object matching in videos. In: ICCV (2003)

2. Lazebnik, S., Schmid, C., Ponce, J.: Beyond bags of features: Spatial pyramid matching for recognizing natural scene categories. In: CVPR (2006)

3. Li, L.-J., Su, H., Xing, E.P., Fei-fei, L.: Object bank: A high-level image representation for scene classification and semantic feature sparsification. In: NIPS (2010)

4. Pandey, M., Lazebnik, S.: Scene recognition and weakly supervised object localization with deformable part-based models. In: ICCV (2011)

5. Quattoni, A., Torralba, A.: Recognizing indoor scenes. In: CVPR (2009)

6. Torralba, A., Fergus, R., Freeman, W.T.: 80 million tiny images: a large database for non-parametric object and scene recognition. PAMI (2008)

7. Hays, J., Efros, A.A.: im2gps: estimating geographic information from a single image. In: CVPR (2008)

8. Ullman, S., Vidal-Naquet, M., Sali, E.: Visual features of intermediate complexity and their use in classification. Nature America (2002)

9. Leung, T., Malik, J.: Representing and recognizing the visual appearance of materials using three-dimensional textons (2001)

10. Brown, M., Szeliski, R., Winder, S.: Multi-image matching using multi-scale oriented patches. In: CVPR (2005)

11. Berg, A.C., Malik, J.: Geometric blur for template matching. In: CVPR (2001)

12. Lowe, D.: Distinctive image features from scale-invariant keypoints. IJCV (2004)

13. Dalal, N., Triggs, B.: Histograms of oriented gradients for human detection. In: CVPR (2005)

14. Felzenszwalb, P., McAllester, D., Ramanan, D.: A discriminatively trained, multiscale, deformable part model. In: CVPR (2008) 
15. Torresani, L., Szummer, M., Fitzgibbon, A.: Efficient Object Category Recognition Using Classemes. In: Daniilidis, K., Maragos, P., Paragios, N. (eds.) ECCV 2010, Part I. LNCS, vol. 6311, pp. 776-789. Springer, Heidelberg (2010)

16. Payet, N., Todorovic, S.: Scene shape from texture of objects. In: CVPR (2011)

17. Bourdev, L., Malik, J.: Poselets: Body part detectors trained using $3 d$ human pose annotations. In: ICCV (2009)

18. Farhadi, A., Endres, I., Hoiem, D.: Attribute-centric recognition for cross-category generalization. In: CVPR (2010)

19. Sadeghi, M.A., Farhadi, A.: Recognition using visual phrases. In: CVPR (2011)

20. Shotton, J., Winn, J.M., Rother, C., Criminisi, A.: TextonBoost: Joint Appearance, Shape and Context Modeling for Multi-class Object Recognition and Segmentation. In: Leonardis, A., Bischof, H., Pinz, A. (eds.) ECCV 2006. LNCS, vol. 3951, pp. 1-15. Springer, Heidelberg (2006)

21. Choi, M.J., Lim, J.J., Torralba, A., Willsky, A.S.: Exploiting hierarchical context on a large database of object categories. In: CVPR (2010)

22. Yao, B., Fei-Fei, L.: Grouplet: A structured image representation for recognizing human and object interactions. In: CVPR (2010)

23. Fergus, R., Perona, P., Zisserman, A.: Object class recognition by unsupervised scale-invariant learning. In: CVPR (2003)

24. Russell, B.C., Freeman, W.T., Efros, A.A., Sivic, J., Zisserman, A.: Using multiple segmentations to discover objects and their extent in image collections. In: CVPR (2006)

25. Todorovic, S., Ahuja, N.: Unsupervised category modeling, recognition, and segmentation in images. PAMI (2008)

26. Kim, G., Faloutsos, C., Hebert, M.: Unsupervised Modeling of Object Categories Using Link Analysis Techniques. In: CVPR (2008)

27. Lee, Y.J., Grauman, K.: Foreground focus: Unsupervised learning from partially matching images. IJCV (2009)

28. Lee, Y.J., Grauman, K.: Object-graphs for context-aware category discovery. In: CVPR (2010)

29. Lee, Y.J., Grauman, K.: Learning the easy things first: Self-paced visual category discovery. In: CVPR (2011)

30. Kim, G., Torralba, A.: Unsupervised Detection of Regions of Interest using Iterative Link Analysis. In: NIPS (2009)

31. Kang, H., Hebert, M., Kanade, T.: Discovering object instances from scenes of daily living. In: ICCV (2011)

32. Shrivastava, A., Malisiewicz, T., Gupta, A., Efros, A.A.: Data-driven visual similarity for cross-domain image matching. ACM ToG (SIGGRAPH Asia) (2011)

33. Ye, J., Zhao, Z., Wu, M.: Discriminative k-means for clustering. In: NIPS (2007)

34. Sivic, J., Russell, B.C., Efros, A.A., Zisserman, A., Freeman, W.T.: Discovering object categories in image collections. In: ICCV (2005)

35. Karlinsky, L., Dinerstein, M., Ullman, S.: Unsupervised feature optimization (ufo): Simultaneous selection of multiple features with their detection parameters. In: CVPR (2009)

36. Everingham, M., Gool, L.V., Williams, C.K.I., Winn, J., Zisserman, A.: The PASCAL Visual Object Classes Challenge (2007)

37. Zhu, J., Li, L.-J., Li, F.-F., Xing, E.P.: Large margin learning of upstream scene understanding models. In: NIPS (2010)

38. Doersch, C., Singh, S., Gupta, A., Sivic, J., Efros, A.A.: What makes paris look like paris? ACM Transactions on Graphics (SIGGRAPH) 31 (2012) 